\title{
EEG/MEG Source Localization Using Source Deflated Matching Pursuit
}

\author{
Shun Chi Wu and A. Lee Swindlehurst
}

\begin{abstract}
A matching pursuit (MP) based algorithm, called source deflated matching pursuit (SDMP), is proposed for locating sources of brain activity. By iteratively deflating the contribution of identified sources to multiple measurement vectors (MMVs), the SDMP algorithm transforms the original multi-basis-vector/matrix selection problem into a single-basisvector/matrix selection problem, which not only mitigates the residual-source interference but also remedies the intrinsic bias when locating deep sources. The robustness of the proposed algorithm to two bias factors is verified through simulations.
\end{abstract}

\section{INTRODUCTION}

Source reconstruction using multi-sensor measurements of electroencephalography (EEG) or magnetoencephalography (MEG) arising from current dipoles located throughout the brain has been studied for decades. It not only retains the high-temporal-resolution characteristic of EEG/MEG but also improves the resolution of reconstructed spatial spectra. Generally, the reconstruction process involves a high dimensional inverse problem that has an infinite number of solutions; to have an appropriate solution, constraints must be incorporated into the problem.

The most well-known constraint in the literature is the "minimum norm" constraint [1], that finds the solution vectors that best match the measurements with the smallest $l_{2}$ norm. However, this approach usually produces overly distributed or smeared sources in the reconstruction region that diverge from the sparse and highly localized nature of brain activity. As a result, researchers have resorted to a "sparseness" constraint [2], [3]. By providing an over-complete dictionary for representing solution vectors, a reconstruction problem with the sparseness constraint will search for a solution vector that not only matches the measurements, but also has as few non-zero entries as possible. This process is also referred to as subspace/basis selection in the literature and typically requires a combinatorial search [4], [3].

To avoid an exhaustive search for optimal solutions, some low-complexity algorithms have been proposed. Matching pursuit (MP) search techniques [5], [6], [7] sequentially rather than simultaneously add basis vectors into the set for representing measurements and then remove their contribution from the measurements. These approaches significantly reduce the search effort since the original multi-

Shun Chi Wu is with the Department of Electrical Engineering and Computer Science, University of California, Irvine, CA 92697 USA scwu@uci.edu

A. Lee Swindlehurst is with the Department of Electrical Engineering and Computer Science, University of California, Irvine, CA 92697 USA swindle@uci.edu basis-vector/matrix selection problem is replaced with several smaller problems where only a basis vector/matrix that best matches the residual measurements is sought for. Diversity measure minimization techniques on the other hand select all the vectors/matrices of the dictionary initially and eliminate the vectors/matrices one-by-one until a requisite number remains [3], [5]. FOCal Underdetermined System Solver (FOCUSS) [2] is a famous example belonging to this algorithm category, which uses a weighted minimum norm approach for sequentially reinforcing strong sources and reducing weak ones and ultimately leads to a sparse solution. Although it is sensitive to noise and highly dependent on the initial estimates [1], there exist some extended FOCUSS algorithms based on other diversity measures (e.g., the $l_{p}$ norm with $p \leq 1$ ) discussed in [8], [3], [5], which can be used for basis selection problems when the signal-to-noise ratio (SNR) is low. However, the cost functions of the diversity measure minimization techniques might not be continuous and a need for a more tractable cost function or a more sophisicated minimization technique is inevitable.

In this paper, we consider application of the MP algorithms to the inverse problem with emphasis on locating sources of EEG or MEG brain activity. When implementing the algorithms, we utilize multiple measurement vectors (MMVs) which arise naturally when measuring stimulusevoked activity [7], [9]. Two factors that will bias the position estimates are presented and a novel MP-based algorithm that can cope with the these bias factors is proposed later.

\section{DATA MODEL AND ASSUMPTIONS}

\section{A. Data Model}

Although from a mathematical standpoint, the techniques we develop are applicable to both EEG and MEG source localization, we develop the model here only for the EEG case. In the standard data model for EEG source localization in the brain, the cortex or brain region of interest is divided into $n$ small disjoint candidate locations, and sources in activated locations are modeled as equivalent current dipoles [10], [11]. The linear forward model relating the moment vector $\mathbf{s}(t) \in \mathbb{R}^{3 n \times 1}$, which is the stack of dipole moments at all candidate regions, and the $m$-sensor array measurements $\mathbf{x}(t) \in \mathbb{R}^{m \times 1}$ at time instant $t$ can be written as

$$
\mathbf{x}(t)=\mathbf{G s}(t)+\mathbf{n}(t)
$$

where $\mathbf{G}=\left[\mathbf{G}\left(\mathbf{r}_{1}\right), \ldots, \mathbf{G}\left(\mathbf{r}_{n}\right)\right] \in \mathbb{R}^{m \times 3 n}$ is the basis matrix that is comprised of the lead field matrices (LFMs) of all $n$ candidate regions, $\mathbf{r}_{k} \in \mathbb{R}^{3 \times 1}$ represents the position of 
the $k^{t h}$ candidate region and $\mathbf{n}(t) \in \mathbb{R}^{m \times 1}$ is the background electronic/neural noise. The LFM G(r) associated with each location $\mathbf{r}$ has three columns, each corresponding to the array response for the dipole moment along the three coordinate axes. Due to the sparse and highly localized nature of the brain activity, most components of the moment vector $\mathbf{s}(t)$ are zero.

As mentioned above, the LFM $\mathbf{G}\left(\mathbf{r}_{k}\right)$ is decomposed into three parts (cf. two components for MEG applications), each due to a separate dipole orientation component:

$$
\mathbf{G}\left(\mathbf{r}_{k}\right)=\left[\mathbf{g}_{x}\left(\mathbf{r}_{k}\right) \mathbf{g}_{y}\left(\mathbf{r}_{k}\right) \mathbf{g}_{z}\left(\mathbf{r}_{k}\right)\right]
$$

where $\mathbf{g}_{x}\left(\mathbf{r}_{k}\right), \mathbf{g}_{y}\left(\mathbf{r}_{k}\right)$ and $\mathbf{g}_{z}\left(\mathbf{r}_{k}\right)$ are the $m \times 1$ lead field vectors (LFVs) that result from unit amplitude sources at $\mathbf{r}_{k}$ and oriented in the $\mathrm{x}, \mathrm{y}$ and $\mathrm{z}$ directions respectively. Hence, the number of columns of the basis matrix $G$ and the number of moment components are 3 times the number of candidate regions. In practice, these LFV's can be derived from multilayer spherical head models [11], [12] or based on realistic head measurements taken from computed tomography or magnetic resonance imaging data [13].

Once multiple measurement vectors (MMVs) are provided (i.e. $t=1, \ldots, T$ with $T>1$ ), the MMV data model can be succinctly written as

$$
\mathbf{X}=\mathbf{G S}+\mathbf{N}
$$

where $\mathbf{X}=[\mathbf{x}(1), \ldots, \mathbf{x}(T)]$, and $\mathbf{S}, \mathbf{N}$ are defined similarly.

\section{B. Assumptions}

We adopt assumptions similar to those made in [7]:

A1 The moment vectors $\mathbf{s}(t)$ for $t=1, \ldots, T$, are sparse, i.e., most of the entries are zero.

A2 The moment vectors $\mathbf{s}(t)$ for $t=1, \ldots, T$, have the same sparsity profile so that the indices of the nonzero entries are independent of $t$.

\section{EXISTING MATCHING PURSUIT BASED TECHNIQUES AND BIAS FACTORS}

The highly localized nature of the evoked brain activity results in highly sparse moment vectors once an over-complete basis matrix is adopted. It means that only limited columns of the basis matrix $\mathbf{G}$ will take effect on the measurements. In general, optimal selection of the lead field matrices that best represent the measurements requires a combinatorial search. However, the cost of such a search may be prohibitive even for a moderate number of sources [3].

By sequentially rather than simultaneously selecting basis vectors into the set for representing the measurement matrix and removing their contribution once determined, MP-based search techniques such as basic matching pursuit (BMP) and order recursive matching pursuit (ORMP) (refer to e.g. [7] for details) can substantially reduce the search effort. This is because they replace the original multi-basis-vector/matrix selection problem with several smaller problems where only a basis vector/matrix that best matches the residual measurements is sought for.

There are two factors that bias the position estimates when using BMP and ORMP for souorce localization. The residual-source interference problem [14] occurs whenever more than one candidate region is simultaneously activated. If $r$ activated regions are present in response to some stimuli, the spatial spectrum generated by the cost function of BMP or ORMP at the $p^{\text {th }}$ iteration will ideally have a single global maximum and at most $r-p-1$ local maxima, and the candidate region corresponding to the global maximum is the target to be selected. However, because of the spatial interferences among sources, the formation of the global maximum is not solely due to the LFM of some specific candidate region; instead, it might be a "compromise" between all $r-p$ residual sources, which will lead to biased estimates. This problem is even more apparent when the distance between sources is small. The presence of sources deep within the brain is the other bias factor, which causes the search techniques to favor candidate regions closest to the sensors. This is because the potential fields rapidly fall off with distance from a dipole source, and a source located closer to the sensors can often be received with higher SNR even if its dipole moment is weaker than others farther away from the sensors [2].

\section{SOURCE DEFLATED MATCHING PURSUIT} (SDMP)

Location indices provided by the BMP or ORMP algorithms normally deviate from the actual source positions due mostly to interference among residual sources. When there exists only one activated location, the discrepancy is largely reduced even for the localization of a deep source. Thus, if the original multi-source search can be replaced by a series of unbiased single-source searches, the influence of residual-source interference on localization accuracy can be eliminated. Provided with a set of biased indices obtained either by BMP or ORMP, we assume that all other location indices except the one for which we are conducting a "fine" search for are accurate. We then suppress or "project out" the contribution of these other sources before conducting the fine search. By doing so, there will ideally be only one source left in the bias-suppressed measurements, and the original problem will reduce to having only one source to locate. A similar idea is used in the alternating projection (AP) algorithm for computing the exact maximum likelihood estimates for the directions of multiple sources in passive sensor arrays [15] and was also used in the beamforming (BF) method for multiple dipole sources localization for MEG [16].

The proposed source deflated matching pursuit (SDMP) algorithm is summarized as follows:

\section{Initialization Stage}

Use the BMP or ORMP algorithm to obtain an initial index 
set of source locations:

$$
I=\left\{k_{1}^{0}, k_{2}^{0}, \ldots, k_{r}^{0}\right\} .
$$

\section{Deflation Stage}

Given $\mathbf{G}_{k}, \mathbf{X}$,

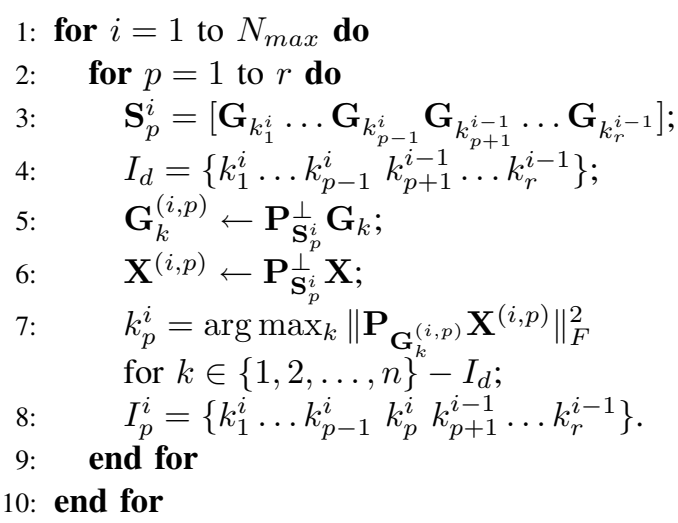

$N_{\max }$ is the maximum allowable number of iterations for the fine search and $r$ is the number of sources we want to locate. The projection matrix $\mathbf{P}_{\mathbf{S}_{p}^{i}}^{\perp}=\mathbf{I}-\mathbf{S}_{p}^{i}\left[\left(\mathbf{S}_{p}^{i}\right)^{T} \mathbf{S}_{p}^{i}\right]^{-1}\left(\mathbf{S}_{p}^{i}\right)^{T}$ is for suppressing the contribution of the sources at locations other than the one for which a fine search is being conducted. Normally, it only takes 2 to 4 iterations to reach a final result where the location indices are no longer updated.

\section{SIMULATION EXAMPLES}

\section{A. Dual-Condition Experiments and Interference Mitigation}

For EEG measurements, the desired signals are very weak, and embedded in strong, spatially correlated interference and noise due primarily to background brain activity not related to the activity of interest. To mitigate the interference, experiments with dual conditions, one (control state) prior to application of the stimulus and one (activity state) after the stimulus are normally adopted [17]. With the implication that both states have statistically similar noise and interference, the control state measurements are used either to estimate the spatial covariance matrix of the interference plus noise for prewhitening the activity state measurements [17] or to construct a subspace that is orthogonal to the spatially correlated interference that can be projected out [12]. Through these preprocessing steps, what is left in the activity state measurements will simply be "modified" task-related signals and white noise.

\section{B. Simulation Parameters}

EEG simulations involving 61 electrodes from the 10-10 system [18] were conducted. The LFM's were calculated using the approximate method of [11], in which the head was modeled as a multi-layer sphere with its radii and conductivities specified in [19]. The brain surface and a brain slice (elevation angle $=30 \mathrm{deg}$ ) were modeled as a spherical shell (upper half) and a flat disk, respectively. The shell and disk were respectively partitioned into 1279 and 676 uniformly distributed voxels as candidate locations, with the average distance between two adjacent locations equal to approximately 0.75 and $0.5 \mathrm{~cm}$, respectively. To generate the received moment matrix GS, the orientation of an activated source dipole was assumed to be constant over the data collection interval. This means that the dipole moments $\mathbf{s}_{k}(t) \in \mathbb{R}^{3 \times 1}$ of a source at $\mathbf{r}_{k}$ can be decomposed as $\mathbf{s}_{k}(t)=\psi s_{k}^{\prime}(t)$ with $\psi \in \mathbb{R}^{3 \times 1}$ denoting the dipole orientation and the scalar time series $s_{k}^{\prime}(t)$ denoting its moment magnitude at time instant $t$. White noise was added to the received moment matrix with its power being specified by a given signal to noise ratio (SNR), defined as $\|\mathbf{G S}\|_{F}^{2} /\|\mathbf{N}\|_{F}^{2}$. We assumed that the spatially correlated background interference had been effectively suppressed by either prewhitening or null projection.

\section{Performance Measures}

To measure the performance of the algorithms, the "success rate" and RMS position estimation error were used. For a given source position $\mathbf{r}_{S}$ and an estimated position $\mathbf{r}_{E}$, we claim a success if $\left\|\mathbf{r}_{S}-\mathbf{r}_{E}\right\| \leq d_{t h}$, where $d_{t h}$ is a threshold distance. The RMS position estimation error is defined as $L o c_{E r r}=\sqrt{\frac{1}{r} \sum_{n=1}^{r}\left\|\mathbf{r}_{S}-\mathbf{r}_{E}\right\|_{F}^{2}}$.

\section{Results and Discussion}

Evoked response potentials (ERPs) arising from different parts of the cerebral cortex are closely related to the brain functions that these parts of the cortex are responsible for. For example, the ERPs from the temporal lobes and the occipital lobe can be connected with some auditory and visual stimuli. In the first simulation, we try to locate these ERPs with three MP algorithms. Sources with random orientations were quasi-randomly placed on the brain surface (each pair of sources was separated by at least $5 \mathrm{~cm}$ ). The SNR was set at $5 \mathrm{~dB}$ and 200 trials were separately conducted for cases involving different numbers of sources. As shown in Figure
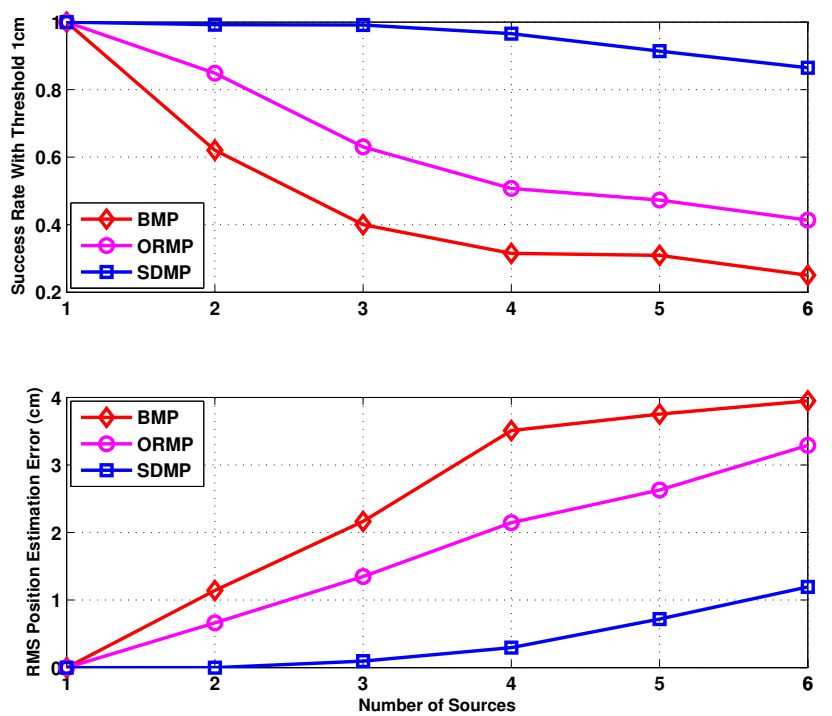

Fig. 1. The influence of the number of sources on localization using three MP algorithms. Upper: success rate with threshold $d_{t h}=1 \mathrm{~cm}$. Lower: RMS position estimation error $(\mathrm{cm})$. 

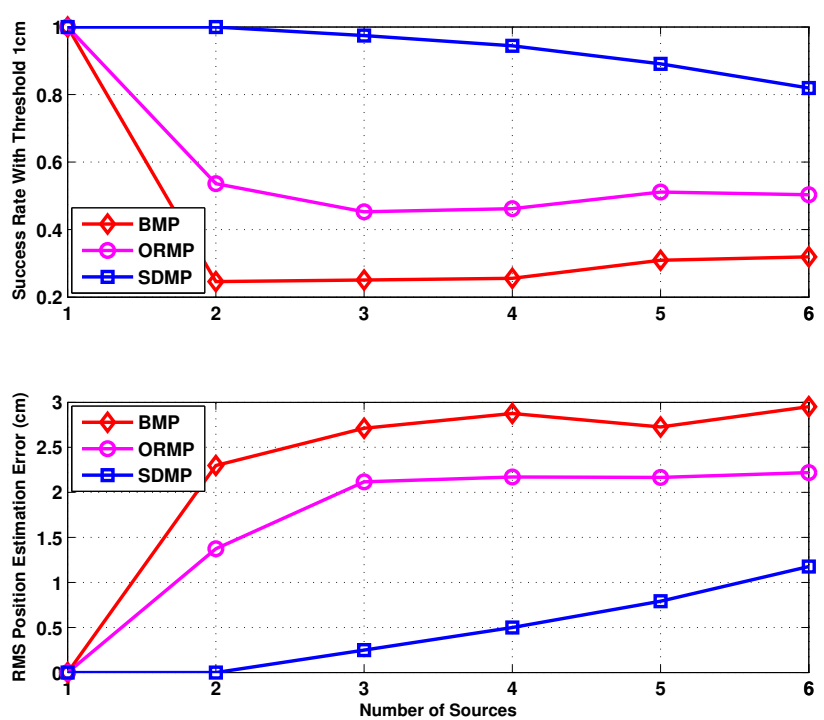

Fig. 2. Deep sources on a brain slice. The influence of the number of sources on localization using three MP algorithms. Upper: success rate with threshold $d_{t h}=1 \mathrm{~cm}$. Lower: RMS position estimation error $(\mathrm{cm})$.

1 , once there exists more than one source, the interference among sources starts taking effect and the performance degrades more and more as the number of sources increases. However, the SDMP algorithm achieves considerably better performance than the BMP and ORMP algorithms in all cases.

One application of EEG source localization is in the field of epilepsy, where it is important to localize the sources of epileptic discharges and study their propagation patterns. For some subjects, the epileptic seizures occur in areas deep inside the brain [20], which causes localization to be more challenging due to the intrinsic bias problem. In the second simulation, the sources were randomly located on the brain slice rather than the brain surface to mimic a case with sources far removed from the sensors. The influence of the number of such sources is plotted in Figure 2 for an SNR of $5 \mathrm{~dB}$. Comparing Figures 1 and 2, we find a further degradation in the performance of both BMP and ORMP algorithms due to the intrinsic bias. As in the first example, when more than one source is present, the performance of both the BMP and ORMP algorithms degrades dramatically. However, the performance of the algorithms tends to be "stable" when more than 2 sources present. This is due to the fact that, although the residual source interference further biases the position estimates obtained by both BMP and ORMP, the sources are confined to the brain slice (a disk) with a radius about $6.8 \mathrm{~cm}$, which limits the size of the errors compared with the first example. However, as before, the SDMP algorithm still maintains significantly better performance in all cases.

\section{CONCLUSIONS}

We have presented a MP based search technique for locating sources of brain activity with MMVs. The proposed algorithm iteratively removes the contribution of identified sources so that the original multi-basis-vector/matrix selection problem can be transformed into a single-basisvector/matrix selection problem, which not only mitigates the residual-source interference but also is immune to the intrinsic bias when locating deep sources. The influence of both bias factors on the performance of existing MP algorithms has been examined and the robustness of the proposed algorithm to these factors has also been verified through simulations.

\section{REFERENCES}

[1] J. W. Phillips, R. M. Leahy, and J. C. Mosher, "MEG-based imaging of focal neuronal current sources," IEEE Transactions on Medical Imaging, vol. 16, pp. 338-348, 1997.

[2] I. F. Gorodnitsky, J. S. George, and B. D. Rao, "Neuromagnetic source imaging with focuss: a recursive weighted minimum norm algorithm," J. Electroenceph. Clinical Neurophysiol., vol. 95, pp. 231-251, 1995.

[3] B. D. Rao, "Signal processing with the sparseness constraint," Proc. IEEE ICASSP, pp. 1861-1864, 1998.

[4] B. K. Natarajan, "Sparse approximate solutions to linear systems," SIAM J. Comput., vol. 24, pp. 227-234, 1995.

[5] B. D. Rao, K. Engan, S. F. Cotter, J. Palmer, and K. Kreutz-delgado, "Subset selection in noise based on diversity measure minimization," IEEE Trans. Signal Processing, vol. 51, pp. 760-769, 2003.

[6] S. F. Cotter, J. Adler, B. D. Rao, and K. Kreutz-Delgado, "Forward sequential algorithms for best basis selection," Proc. Inst. Elect. Eng. Vision, Image, Signal Process., vol. 146, pp. 235-244, 1999.

[7] S. F. Cotter, B. D. Rao, K. Engan, and K. Kreutz-Delgado, "Sparse solutions to linear inverse problems with multiple measurement vectors," IEEE Transactions on Signal Processing, vol. 53, pp. 24772488,2005

[8] K. Kreuta-Delgado and B. Rao, "Measures and algorithms for best basis selection," Proc. IEEE ICASSP, pp. 1881-1884, 1998.

[9] Z. Zhang and B. Rao, "Sparse signal recovery in the presence of correlated multiple measurement vectors," Proc. IEEE ICASSP, pp. 3986-3989, 2010.

[10] J. Sarvas, "Basic mathematical and electromagnetic concepts of the biomagnetic inverse problem," Phys. Med. Biol., vol. 32, pp. 11-22, 1987.

[11] M. Sun, "An efficient algorithm for computing multi-shell spherical volume conductor models in EEG dipole source localization," IEEE Trans. Biomed. Eng., vol. 44, pp. 1243-1252, 1997.

[12] S. C. Wu, A. L. Swindlehurst, and Y. C. Yao, "Direct interference suppression in eeg/meg dipole source localization," Proc. IEEE ICASSP, pp. 574-577, 2010.

[13] B. N. Cuffin, "EEG localization accuracy improvements using realistically shaped head models," IEEE Trans. Biomed. Eng., vol. 43, pp. 299-303, 1996.

[14] S. K. Oh and C. K. Un, "A sequential estimation approach for performance improvement of eigenstructure-based methods in array processing," IEEE Trans. Signal Processing, vol. 41, pp. 457-463, 1993.

[15] I. Ziskind and M. Wax, "Maximum likelihood localization of multiple sources by alternating projection," IEEE Trans. Acoust., Speech and Signal Processing, vol. 36, pp. 1553-1560, 1988.

[16] H. Mohseni and S. Sanei, "A new beamforming-based meg dipole source localization method," Proc. IEEE ICASSP, pp. 558-561, 2010.

[17] K. Sekihara, K. Hild, and S. Nagarajan, "A novel adaptive beamformer for MEG source reconstruction effective when large background brain activities exist," IEEE Trans. Biomed. Eng., vol. 53, pp. 1755-1764, 2006.

[18] R. Oostenveld and P. Praamstra, "The five percent electrode system for high-resolution EEG and ERP measurements," Clin. Neurophysiol., vol. 112, pp. 713-719, 2001.

[19] B. N. Cuffin and D. Cohen, "Comparison of the magnetoencephalogram and electroencephalogram," Electroencephalogr. Clin. Neurophysiol., vol. 47, pp. 132-146, 1979.

[20] J. Dauwels, E. Eskandar, and S. Cash, "Localization of seizure onset area from intracranial non-seizure eeg by exploiting locally enhanced synchrony," Proc. IEEE EMBS, pp. 2180-2183, 2009. 